\title{
Intracranial self-stimulation and escape by EEG-derived instrumental responses in cerveau isolé rats
}

\author{
JAMES J. KEENE and NILDA M. KEENE \\ Department of Physiology, University of Puerto Rico School of Medicine, San Juan, Puerto Rico 00936
}

\begin{abstract}
In 14 of the 25 unanesthetized postcollicular, pretrigeminal cerveau isolé rats studied, intracranial self-stimulation or escape, or both, were demonstrated using EEG-derived instrumental responses. Stimulus trains $(0.5$-sec, 40 -cps trains of 0.5 -msec cathodal pulses at $600 \mu \mathrm{A})$ were delivered in a continuous reinforcement schedule in series of tests for each of 55 diencephalic and mesencephalic sites. Medial forebrain bundle stimulation consistently elicited self-stimulation, but never evoked escape. Stimulation of known aversive regions, lateral reticular formation, medial lemniscus, and meninges, elicited escape, but not self-stimulation. Both self-stimulation and escape were obtained by two types of EEG responses: high and low voltage, which triggered reinforcements when EEG amplitude increased and decreased, respectively. These data extend previous concepts of the integrative capability of cerveau isolé animals to include phenomena related to reward, pain, and neural plasticity. The cerveau isolé technique presented may offer significant advantages for several types of experiments for which the paralyzed encephalé isolé preparation has been the traditional choice.
\end{abstract}

This paper presents evidence of intracranial selfstimulation and escape in unanesthetized cerveau isole rats, where the operant response was derived from the cortical EEG. The possible significance of this demonstration is reflected by the use of behavioral terms to summarize the effects observed. That is, the evidence implies that phenomena related to reward, pain, and learning or plasticity, can occur in the isolated forebrain with midbrain. Much of the detail of this report bears on the issue of whether self-stimulation and escape defined by EEG responses in the cerveau isolé preparation warrant description by the behavioral concepts invoked.

Our present interest originated from recent reports (Keene, 1973a, b; 1975c) which have proposed a physiological definition and anatomical localization of affect coding by single neurons, based on prolonged opposite responses, increased vs. decreased firing rates, to believed rewarding and aversive brain stimulation. These unit responses lasted seconds and were anatomically confined to intralaminar thalamus and medial pallidum. These data were obtained from the same cerveau isolé preparation used in the present study, and their behavioral interpretation in terms of affect coding called for confirmation in intact animals. This was done in awake, chronically implanted cats, in which single-cell recordings showed the suggested affect-related responsiveness to lateral hypothalamic and midbrain reticular stimulation,

The authors wish to thank Angeles Chaves for assistance in neurohistology. confirmed to be rewarding and aversive, respectively, by behavioral tests in the same animal (Keene, 1975a). If the affect coding proposal withstood this test in chronic, intact animals, another inference is that rewarding and aversive properties of brain stimulation might be demonstrable in the cerveau isolé animal. Thus, the present study sought such effects in this preparation, with the view that they would tend to support the previous interpretation of unit responses as affect-related.

Demonstration of apparent reward and pain effects in the cerveau isolé animal may not be surprising in view of the classical literature on this preparation (Bremer, 1935; Moruzzi, 1964), which has been concerned mostly with the effects of brain transection at various levels on EEG and ocular signs of arousal or wakefulness. When the postcollicular transection is performed on cats, spindles predominate in the EEG with some periods of lowvoltage fast activity, but without clear ocular signs of wakefulness. In the present study using rats, the transections were both postcollicular and pretrigeminal. In the midpontine pretrigeminal cat, periods of EEG synchronization and desynchronization alternate; the desynchronization is more prolonged than in the higher postcollicular section, and is accompanied by ocular signs of wakefulness. It is reasonable to suppose, then, that cerveau isolé animals such as these might be able to manipulate aspects of their EEG as an instrumental response to either increase or decrease the delivery of intracranial reinforcements of positive or negative motivational valence, respectively. 


\section{METHODS}

The postcollicular, pretrigeminal cerveau isolé preparation used in this lab has been described in previous reports (Keene, $1973 \mathrm{a}$ b, 1975b, c). Male and female rats ranging from 250 to $400 \mathrm{~g}$ were used. Under ether or Brevital anesthesia, a centered transverse slit, approximately $1 \times 7 \mathrm{~mm}$, was drilled as far posteriorly as possible on the dorsal surface of the skull. After cutting the dura, the section was made with a scalpel blade, specially shaped to the ventral contour of the midbrain-hindbrain junction. The ventral cutting edge was ground to a taper, but not sharp, and notched in the middle so the basilar artery would be spared. This blade was passed through the skull slit, in an anterior-ventral direction approximately $35 \mathrm{deg}$ from vertical with the bregma-lambda line horizontal, until the ventral surface of the cranium was felt after an approximately 12 -mm excursion from the skull surface. The slit was sealed with bone wax and no further anesthesia was used in the spontaneously respiring preparation. This procedure resulted in little blood loss; indeed, hemorrhage was often visible at the skull surface only when the dura was cut before the section. Rectal temperature, sensed by a thermistor, was automatically maintained as necessary at $38.5^{\circ} \mathrm{C}$ by a heat pad.

In each animal, a twisted pair of 0.2-mm-diam nichrome wires, exposed only at the cross-section of the tips, was placed in the lateral hypothalamic area, and another pair, in the midbrain. In some rats, when these initial stimulating sites had been tested, the stimulating electrodes were raised in $1-\mathrm{mm}$ steps, one or more times, to test additional sites. In all, 55 stimulation sites were tested in 25 rats (Figure 1).

An electrocorticogram (1-50 cps), which will be called the EEG, was recorded differentially from two $0.25-\mathrm{mm}$ stainless steel wires with $1-\mathrm{mm}$ exposed tips, placed bilaterally, $3 \mathrm{~mm}$ anterior to bregma and $2.5 \mathrm{~mm}$ lateral, $2 \mathrm{~mm}$ below the skull surface, and shielded to the skull surface. These locations are on the border between areas 10 and 6 (Skinner, 1971). The average peak-to-peak amplitude of the spontaneous EEG was 100-200 $\mu \mathrm{V}$. The EEG recording was similar, and displayed at the same gain (except in Figure 2), in all of the preparations.

Two procedures were used to derive "instrumental responses" from the EEG recording. In the first group of 32 sites tested in 14 rats, standard pulses were generated by a differential comparator and one-shot when the adjustable comparator level was in the range of the recording. These pulses were counted by a binary counter which was reset at $1-\mathrm{sec}$ intervals. A response was defined as a count of 8 or more pulses in $1 \mathrm{sec}$. This response will be called "high voltage," since, in practice, it was accomplished mostly by increased EEG amplitude (Figure 2). In the second group of 11 rats, where 23 sites were tested, similar digital logic was used to define a roughly opposite "low-voltage" response,
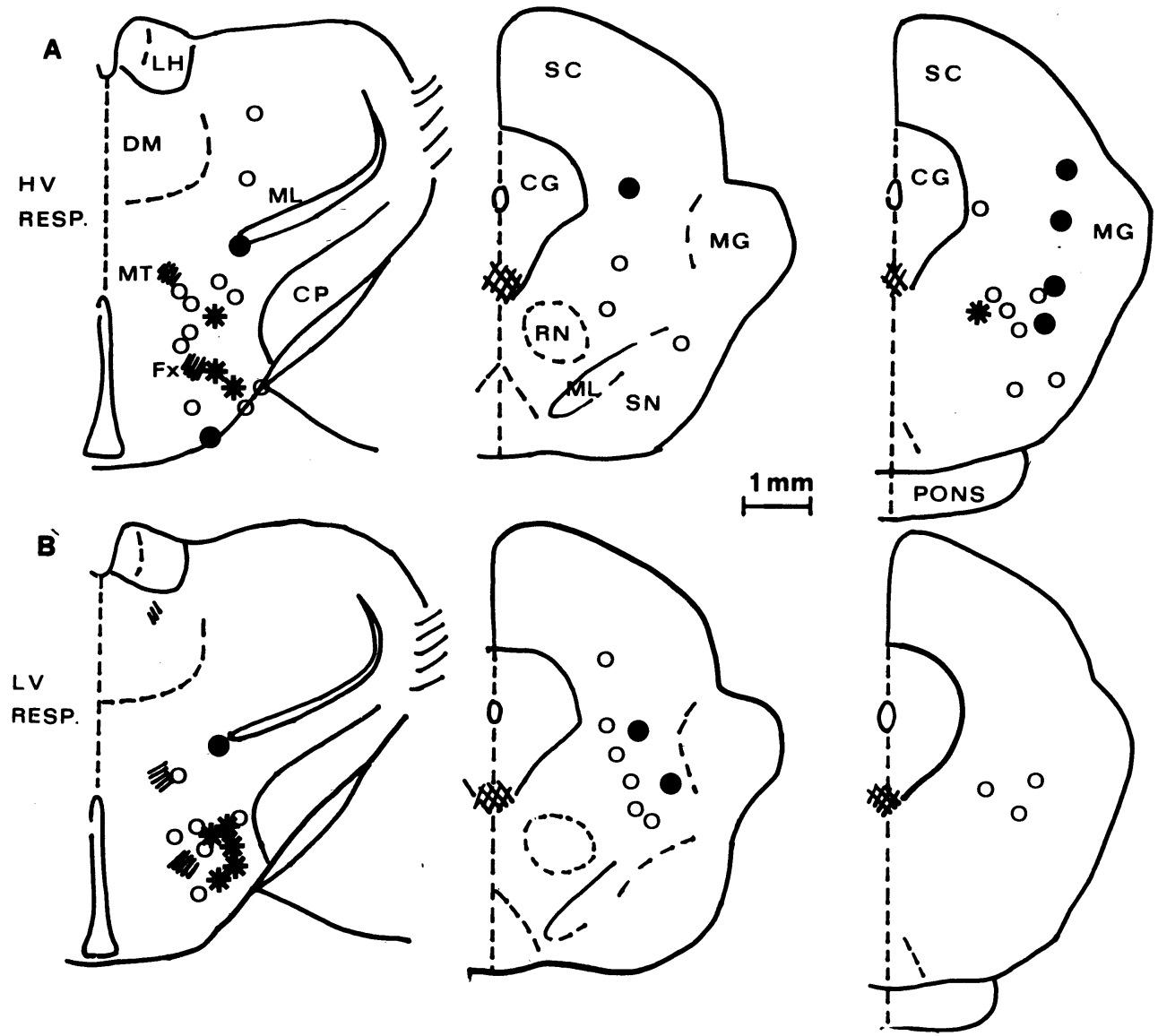

Figure 1. Self-stimulation (asterisks), escape (dots), and neutral sites (circles) defined by stimuluscontingent changes in two types of EEG-derived instrumental responses: a high-voltage ( $\mathbf{H V}$ ) response (A) and a low-voltage (LV) response (B). The sections shown are perpendicular to the bregma-lambda line and are approximately 3.5 (left), 6.0 (center), and 7.0 (right) $\mathrm{mm}$ posterior to bregma. Abbreviations: LH, lateral habenula; DM, dorsal medial nucleus, ML, medial lemniscus; MT, mammillothalamic tract; Fx, fornix; CP, cerebral peduncle; SC, superior colliculus; CG, central gray; MG, medial geniculate; $R N$, red nucleus; $S N$, substantia nigra. 


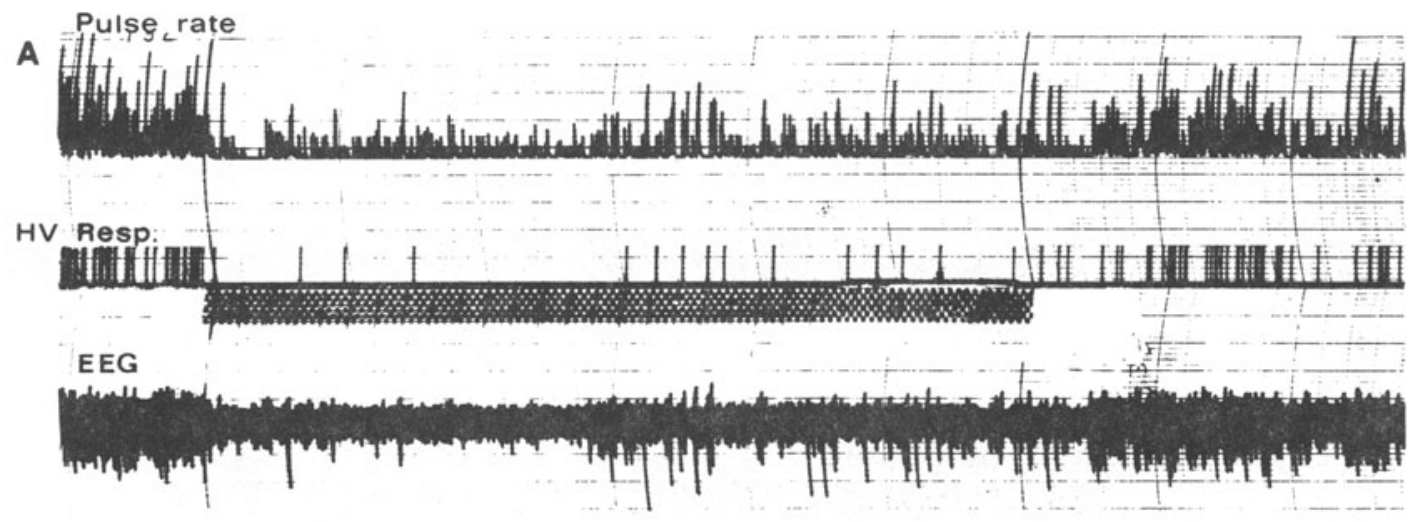

B

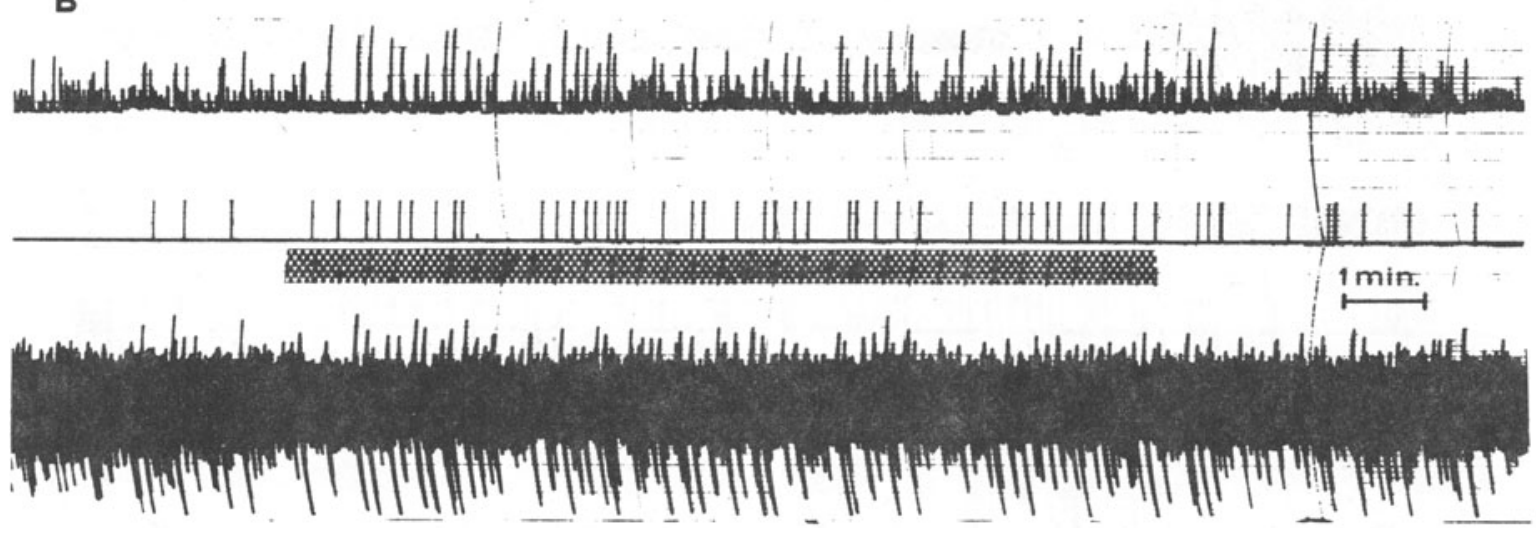

Figure 2. Typical records of escape $(A)$ and self-stimulation $(B)$ defined by rate changes in an EEG-derived high-voltage (HV) response during periods (dotted bars) where brain stimulation was delivered on a continuous reinforcement schedule. Note the relation between the pulse rate and the response rate, and the apparent "extinction" periods following the reinforcement periods.

which occurred when less than 8 pulses were generated in $1 \mathrm{sec}$ (Figures 3 and 4). For both the high- and low-voltage responses, the maximum possible rate was $1 / \mathrm{sec}$, since the responses were defined in terms of 1 -sec intervals. The integrated pulse rate, pulses representing responses, and the EEG were displayed on a polygraph. The positive peaks (up) of the EEG records (Figures $2-4$ ) were slightly attenuated by the comparator load. Responses were also tabulated by an electromechanical counter.

After completion of the preparation, which required less than $30 \mathrm{~min}$ from the administration of anesthesia, a waiting period of 53 to 247 min (mean and SEM, 112 \pm 8 ) elapsed before testing to allow recovery from the anesthesia and spreading depression that was sometimes caused by the surgical procedures. The shorter waiting periods occurred in the animals where ether was used. Toward the end of the waiting period, the comparator level was set so that a steady rate of responses was produced, the overall average of which was $8.60 \pm 0.52$ (SEM) responses/min. Testing was not begun until the EEG was judged to be normal and the response rate was stable.

Each test consisted of three periods, ranging from 7 to $38 \mathrm{~min}$ each, with an average duration of $16 \mathrm{~min}$ each. The longer test periods were implemented when relative EEG synchronization alternated with desynchronization in 10 - to 15 -min cycles, so that each test period would include two or more cycles. The first and third periods were pre- and postreinforcement, respectively, without brain stimulation. In the second reinforcement period, each response triggered a $0.5-\mathrm{sec}, 40-\mathrm{cps}$ train of $0.5-\mathrm{msec}$, $600-\mu \mathrm{A}$ cathodal pulses, delivered to the site being tested, via a constant current device isolated from ground. In some cases, several minutes elapsed before a significantly changed response rate during reinforcement returned to previously obtained spontaneous rates (e.g., Figure 3B); this "exinction" period was not included in the postreinforcement periods used below to define self-stimulation and escape. Usually, 4 to 6 consecutive tests (range 3 to 9) were run at each site, before other sites were stimulated. In many cases, the third, postreinforcement period served also as the prereinforcement period for the subsequent test.

A site was considered to elicit self-stimulation when the response rate during reinforcement was significantly greater $(p<.05)$ than both the corresponding pre- and postreinforcement rates, according to paired t comparisons. Escape was similarly defined as a significantly decreased rate during reinforcement periods.

At the end of the experiments, the animals were sacrificed with an overdose of pentobarbital and perfused with isotonic saline and $10 \%$ Formalin. Frozen sections of $40 \mu$ were mounted and stained for cells or fibers. All of the stimulation sites were identified and projected on to composite drawings (Figure 1), where greatest weight was given to anatomical landmarks nearest to the stimulation site in cases where the overall size of the section differed significantly from the average.

\section{RESULTS}

According to the all-or-none definition of selfstimulation and escape employed, 9 stimulation sites 

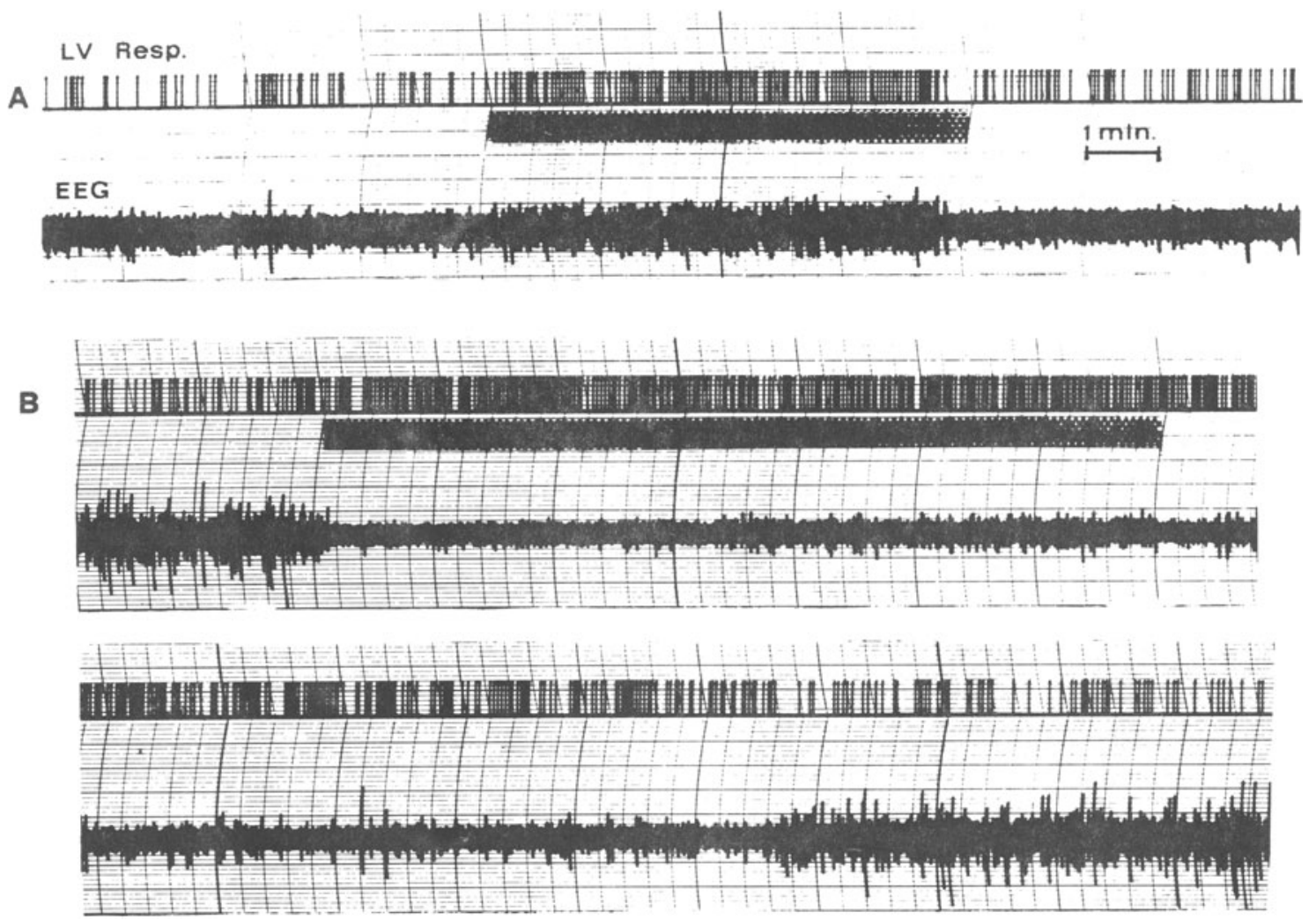

Figure 3. Increased rate of low-voltage (LV) EEG responses, suggesting self-stimulation, during reinforcement periods (dotted bars). (A) The increased response rate during the reinforcement period reflects decreased EEG amplitude, which is obscured by evoked potentials during the stimulus trains. (B) An increased response rate in another rat is maintained for minutes after termination of reinforcements, until it is finally "extinguished," as shown by the continuous records. In this case, the reinforcing trains also elicited evoked potentials of constant wave-form and latency after each stimulus pulse, but their peak-to-peak amplitude was similar to the overall recording.

elicited self-stimulation and 10 elicited escape by means of EEG-derived instrumental responses (Figures 1-5). For 4 of the 9 self-stimulation sites and 7 of the 10 escape sites, these effects were evoked in the group of rats where high-voltage responses were used (Figures $1 \mathrm{~A}$ and 2), with the remainder involving changes in the rate of low-voltage responses (Figures $1 \mathrm{~B}, 3$, and 4$)$. There was not a significant relation $\left(\chi^{2}=0.87\right)$ between the direction of the significant rate changes, self-stimulation or escape, and the direction of the required EEG response, high or low voltage. Both self-stimulation and escape were elicited from stimulation sites in both diencephalon and mesencephalon (Figure 1).

With the exception of one site near ventral medial tegmentum, a region known to elicit behavioral selfstimulation (Routtenberg \& Malsbury, 1969), all of the self-stimulation sites were located in the lateral hypothalamus, in or on the perimeter of the medial forebrain bundle, a well-known positively reinforcing site (Olds \& Olds, 1963). All of the mesencephalic escape sites were in the reticular region, and it appears that dorsal lateral sites, some near the medial geniculate nucleus, were most effective in the present experimental situation. Two of the diencephalic escape sites were near the medial lemniscus, with the third at the ventral border between medial and lateral hypothalamus, penetrating to the meninges. The escape sites were also consistent with behavioral evidence (Olds \& Olds, 1963).

Figure 5 shows the mean responses per minute before, during, and after the reinforcement periods for the three groups of sites: self-stimulation, escape, and neutral. There was a mean increase of $46 \%$ in the response rate during reinforcement periods at the self-stimulation sites; a mean decrease of $50 \%$ in rate occurred at the sites classified as eliciting escape. The SEM's are presented only as estimates of the variability among the sites in self-stimulation and escape performance, since the statistical significance of the rate changes was evaluated on a site-by-site basis.

The data were further studied to determine if any parameters of the experimental procedures might 


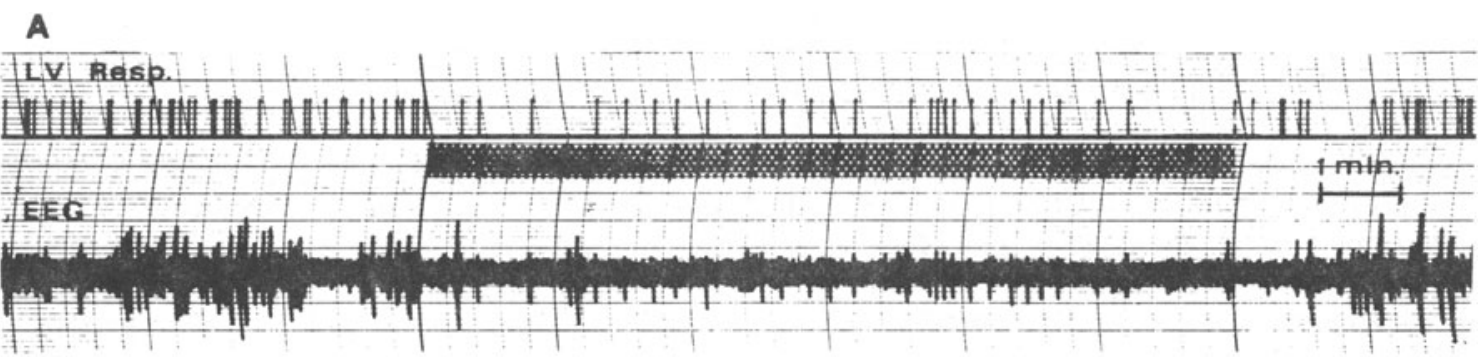

B
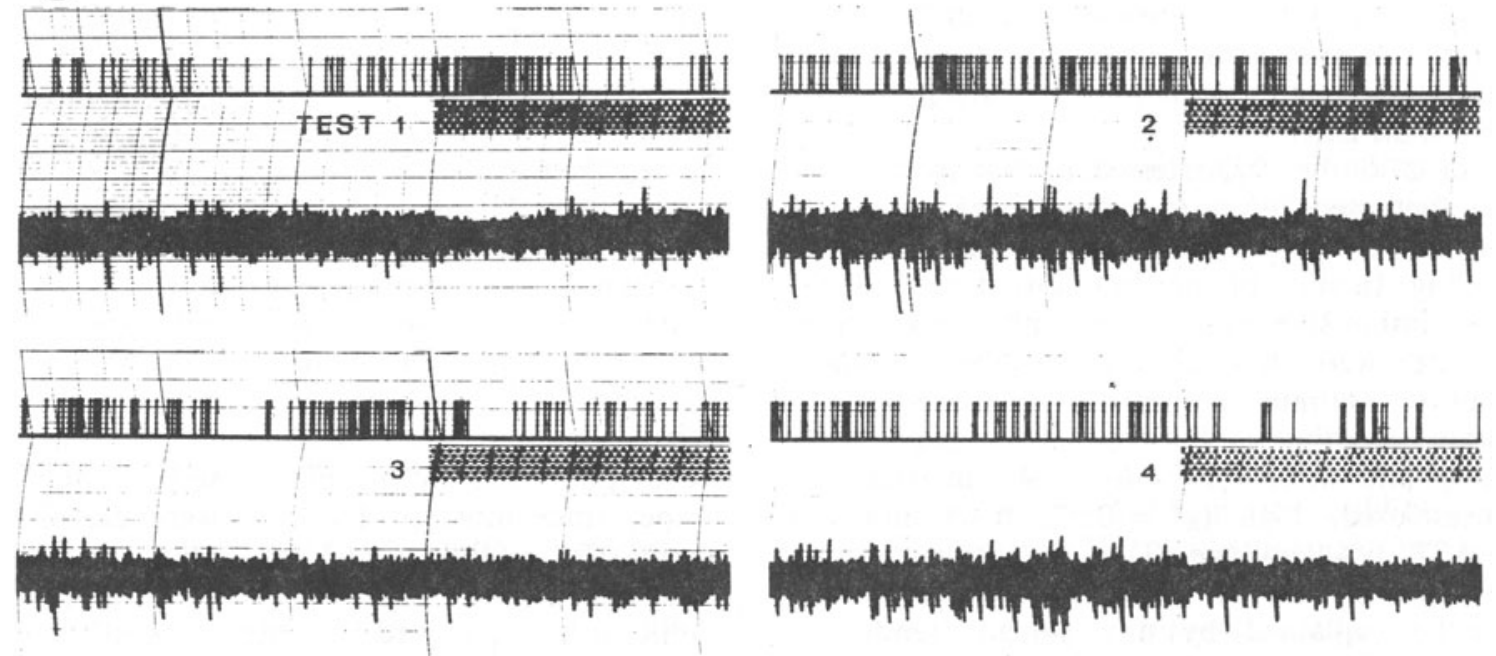

Figure 4. (A) Decreased rate of low-voltage (LV) EEG responses, suggesting escape, during a period (dotted bar) in which each response triggered a 0.5 -sec train of reticular stimulation. (B) Four successive tests showing the beginning of the continuous reinforcement periods (dotted bars) where stimulus trains were delivered to another reticular site in a different rat. When the response-contingent stimulation is first switched on (TEST 1), stimulus-elicited desynchronization generates an increased rate of $L V$ responses. Later in Test 1 , and progressively in Tests 2 through 4 , this positive feedback loop is replaced by decreased responding in the reinforcement periods.

account for the significant response rate changes, other than the anatomical locations of the stimulation sites mapped in Figure 1.

First, considering all three groups of sites, Figure 5 shows that there were no significant differences among the pre- and postreinforcement spontaneous response rates, which might account for the occurrence of self-stimulation or escape.

Second, there were also no significant differences among the groups of sites in the mean waiting periods before testing was begun, or in the average duration of the three periods which comprised each test.

Third, significant variations in the placement of the cerveau isole section were also considered. Gross examination of the brains revealed that the ventral location of the section was most often at the midbrainhindbrain border as marked by the anterior extent of the pons. In some animals, the ventral extent of the section was found to be more anterior, but never involving the mammillary bodies, or slightly posterior

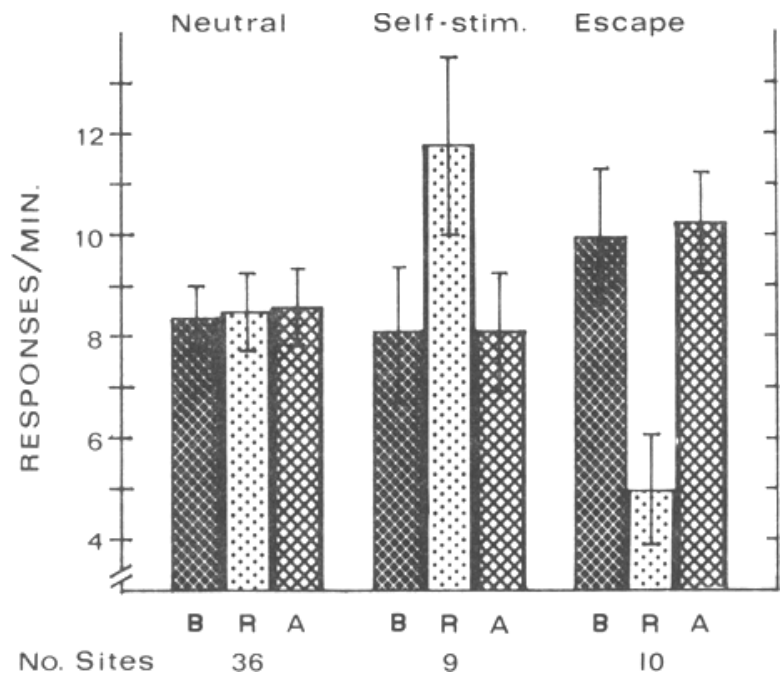

Figure 5. Mean rates of EEG-derived responses for three groups of stimulation sites, neutral, self-stimulation, and escape, before $(B)$, during ( $R)$, and after $(A)$ continuous reinforcement periods. 
to the anterior extent of the pons. No relation was noted, however, between these variations and the observation of self-stimulation or escape. Also, we do not believe that there was significant variation in the completeness of the section. The hindbrain was usually attached to the rest of the brain only by the basilar artery and lateral cerebellar lobes. In some cases, some far lateral brainstem connections had been spared. Likewise, a thin ventral lining of partially crushed fibers were occasionally spared. But the brain stimulation did not elicit movements in these animals. Presumed noxious stimulation to the limbs or head did elicit movements in the otherwise immobile preparations, but was never observed to elicit either evoked potentials or any prolonged changes in the EEG.

Fourth, 27 of the 55 sites tested, or roughly one-half, elicited increased response rates during reinforcement periods, with the remainder eliciting decreased responding. In spite of the finding that most of the self-stimulation sites were in diencephalon and most escape sites were in midbrain, the above random distribution of rate increases and decreases was randomly distributed between diencephalic and mesencephalic sites, for both of the instrumental responses used, high $\left(\chi^{2}=0.57\right.$, n.s. $)$ and low $\left(\chi^{2}=0.77\right.$, n.s. $)$ voltage. That is, the anatomical distribution of self-stimulation and escape sites cannot be explained by any general tendencies revealed in the present sample for either diencephalic or mesencephalic sites to elicit response rate changes in a specific direction, for either the high- or lowvoltage responses.

Fifth, a matrix of product-moment correlations among all of the variables discussed thus far (except the precise location of the cerveau isole section), was computed to determine if there was any significant covariation between these aspects of the experimental procedures and the response rate changes during reinforcement periods, considering all 55 sites. In agreement with the above analysis based on mean values and subgroups of sites, none of the pertinent correlations were statistically significant.

Finally, several additional observations further emphasize the dependence of self-stimulation and escape on the anatomical location of the stimulation site. In two rats, both self-stimulation and escape occurred in the same animal. In both rats, medial forebrain bundle stimulation elicited self-stimulation and midbrain reticular stimulation elicited escape. In one of these animals, the high-voltage response was operative. The inverse changes in EEG amplitude occurred in the other rat, since the low-voltage response was used. Indeed, the discrimination between medial forebrain bundle and midbrain reticular stimulation reflected by the self-stimulation and escape effects was obtained in this animal even though the site of stimulation was alternated in successive tests. In another rat, after an escape effect was established with stimulation of a site near medial lemniscus (Figure 1A), two additional sites were tested with the same electrode, 1 and $2 \mathrm{~mm}$ dorsal to the initial site. Neither of these more dorsal sites in thalamus elicited significant effects.

Several other features of these apparent selfstimulation and escape phenomena deserve mention. One is that, in almost all cases where the effects were elicited, three tests were sufficient to establish the statistical significance of the repeated rate changes, although the power of the paired $t$ comparisons used is substantially diminished with such small Ns. In other words, the effects appeared to be robust and were usually visible, when present, in the first test in a series. Hence, the processes by which significant response rate changes were accomplished could be letected in a matter of minutes and, in some cases, in perhaps less than a minute.

Stimulation at almost all of the sites elicited clear biphasic evoked potentials in the 25 -msec intervals following individual stimulus pulses in the $0.5-\mathrm{sec}$ trains. However, the presence of these constant latency potentials did not predict self-stimulation or escape, since most of the sites were classified as neutral. In addition, self-stimulation and escape were obtained independently of the evoked potential amplitude (e.g., Figure 3). These evoked potentials during stimulus trains could not directly affect the response rate, since only one response could occur per second.

Stimulus trains at many of the sites elicited some degree of EEG desynchronization lasting several seconds. This effect was sometimes opposed to the instrumental responding that occurred when this involved a general increase in EEG amplitude during reinforcement periods (e.g., Figure 4B).

Interposed between the reinforcement and postreinforcement periods in the above analysis, extinction periods were defined in some cases of selfstimulation and escape where a significantly changed response rate was maintained more than several minutes after reinforcement was terminated (e.g., Figure 3B). Over successive tests, the duration of these periods, and the presumed instrumental responding during them, both generally decreased, to the extent that they were often no longer distinguishable in later tests (e.g., Figure 3A). At one selfstimulation site, there was a dramatic, further increase in the high-voltage response rate after reinforcement was withdrawn in the first test, reminiscent of an animal rattling a lever which had triggered reinforcements but which had become inoperative at the beginning of the first extinction period. The brain stimulation during the reinforcement period appeared to have desynchronizing effects on the EEG, and its 
removal would therefore account for this seeming display of frustration. In any case, this effect did not occur in later tests (Figure 2B).

\section{DISCUSSION}

In this report, self-stimulation and escape refer to statistically defined effects analogical to similarly defined behavioral phenomena, for which there were three requirements. First, a given response must change its frequency of occurrence when reinforcement is made contingent on it. Second, this change in response rate should be reversible, "extinguished" when reinforcement is terminated. Third, such presumed control of response rate by reinforcement should occur consistently over repeated periods of reinforcement and extinction. A major result of the present study is that self-stimulation and escape, according to these criteria, were demonstrated for EEG-derived responses in cerveau isolé animals. Control of various features of electrical brain activity by reinforcement procedures has been reported (Fetz, 1969; Fox \& Rudell, 1970; Kamiya, 1969; Olds, 1963; Rosenfeld, 1974). The new result, then, is that this type of phenomenon can occur in the isolated forebrain with midbrain.

The present experiments was designed to test the hypothesis that electrical stimulation of the medial forebrain bundle in the lateral hypothalamus and of the midbrain reticular formation have rewarding and aversive properties, respectively, in cerveau isolé rats. Elicitation of self-stimulation and escape was therefore used to evaluate the motivational properties of stimulus trains, similar to those used in behavioral tests, delivered as reinforcements to 55 anatomical locations. The results provide strong support for the hypothesis. Self-stimulation was obtained at most of the sites in or bordering the medial forebrain bundle, and clear escape performance was elicited by sites, mostly in the reticular formation, which have been shown to be aversive by behavioral methods (Olds \& Olds, 1963). Equally important, self-stimulation was never obtained from sites thought to be strongly aversive, and none of the sites in or near the medial forebrain bundle elicited escape. Most of the ineffective sites, moreover, are thought to be neutral, mixed, or less potent in their motivational effects, on the basis of behavioral data.

The interpretation of the present data in relation to the behaviorally defined concepts of self-stimulation and escape implies that the results cannot be satisfactorily explained in terms of known effects on the EEG of brain stimulation similar to that used in the present experiments. However, EEG desynchronization following high-frequency stimulation of the reticular formation (Moruzzi \& Magoun, 1949) may account for some of the effects obtained. Specifically, stimulus-elicited desynchronization would favor increased rates (self-stimulation) of the low-voltage responses and decreased rates (escape) of the highvoltage responses. On the other hand, such a stimulusbound effect favoring self-stimulation occurred and was reversed, resulting in reduced responding (Figure 4B). Stimulus-elicited desynchronization would not predict escape through decreased low-voltage responses, which was clearly elicited from three stimulation sites (Figure 1B and 4A), excluding the site shown in Figure 4B. Self-stimulation via highvoltage responses would also not be predicted. Indeed, in the test shown in Figure 2B, the EEG amplitude was generally decreased during the reinforcement period, and increased responding was accomplished by the generation of spindles in this context.

In addition, even the cases of self-stimulation via low-voltage responses and escape via high-voltage responses may not be fully explained by desynchronizing effects of the brain stimulation. Compariing the initial and later portions of the reinforcement period in Figure 3B, for example, the increased lowvoltage response rate (self-stimulation) was maintained although higher voltage activity appeared in the later segment. Thus, the arbitrary criteria used to define the low-voltage response could be satisfied without complete EEG desynchronization, and the self-stimulation model suggests, of course, that the animal had "learned" this discrimination. Furthermore, in the escape record in Figure $2 \mathrm{~A}$, it is noteworthy that the EEG is more desynchronized in the first part of the reinforcement period than in the second part, where the number of stimulus trains (responses) is greater, contrary to what might be expected if this escape effect was caused only by stimulus-elicited desynchronization.

Reports of "postreinforcement synchronization" in cortical EEG recordings following a reward presentation (Marczynski, 1972) may also be relevant to the present results, although evoked synchronization following the stimulus trains and lasting more than $1 \mathrm{sec}$ was never observed in polygraph records at faster speeds. Nevertheless, a small, but consistent, synchronizing effect of the stimulation would facilitate self-stimulaton with high-voltage responses and escape with low-voltage responses, but fails to account for either the significant response rate changes in reinforcement periods based on decreased EEG amplitude or the anatomical distribution of effective sites.

The application of a self-stimulation and escape model to the present data, then,. suggests that active, integrative processes are involved. The behavioral analogy may be extended to include the frequent observations of improvement of self-stimulation and escape performance as the reinforcement periods progressed, the apparent suppression of opposing stimulus-elicited responses to achieve criterion per- 
formance, and the acquisition of more efficient extinction in successive tests, reflected by more rapid reversals to prereinforcement rates of responding when reinforcements were switched off.

Finally, it appears that the unanesthetized cerveau isolé preparation might be appropriate for a wide variety of neurophysiological experiments where hindbrain connections with anterior structures are not considered necessary. Indeed, the substantial reduction of sensory input to the forebrain in this preparation may provide favorable conditions for a number of experiments, and in some cases, an alternative to the use of curare to control for proprioceptive feedback, especially when the central effects of this agent may be pertinent. Paralyzed encéphale isolé preparations have been popular for many years among neurophysiologists. The comparative advantages of the simplicity of the preparation and maintenance of a cerveau isolé animal should not be underestimated, considering that artificial respiration and local and general anesthesia are not required after the section. The present method of sectioning has been used on cats in another study in progress in this lab, where a specially shaped blade was inserted posterior to, and roughly following the course of, the tentorium. All four preparations completed thus far were successful and remained in good condition, as judged by the EEG and single-cell responses to brain stimulation, for $24 \mathrm{~h}$ or more, when the experiments were terminated.

\section{REFERENCES}

Bremer, F. Cerveau isolé et physiologie du sommeil. Comptes Rendus de la Societé de Biologie, 1935, 118, 1235-1241.

FETZ, E. E. Operant conditioning of cortical unit activity. Science, $1969,163,955-958$.

Fox, S. S., \& Rudell, A. P. Operant controlled neural event: Functional independence in behavioral coding by early and late components of visual cortical evoked response in cats. Journal of Neurophysiology, 1970, 33, 548-561.

KamiYA, J. Operant control of the EEG alpha rhythm and some of its reported effects on consciousness. In C. Tart (Ed.), Altered states of consciousness. New York: Wiley, 1969. Pp. 507-517.

KeENE, J. J. Opposite medial thalamic unit responses to rewarding and aversive brain stimulation. Experimental Neurology, 1973, 39, 19-35. (a)

KeENE, J. J. Reward-associated inhibition and pain-associated excitation lasting seconds in single intralaminar thalamic units. Brain Research, 1973, 64, 211-224. (b)

KEENE, J. J. Model of affect coding by intralaminar thalamic neurons: Supporting evidence. Society for Neuroscience, 1975, 5, 483. (a)

Keene, J. J. Prolonged unit responses in thalamic reticular, ventral, and posterior nuclei following lateral hypothalamic and midbrain reticular stimulation. Journal of Neuroscience Research, 1975, 1, 459-469. (b)

KEENE, J. J. Reward-associated excitation and pain-associated inhibition lasting seconds in rat medial pallidal units. Experimental Neurology, 1975, 49, 97-114. (c)

MARCZYNSKI, T. J. Electrophysiological correlates of positive reinforcement: Post-reinforcement synchronization, modulation of sensory input, and steady potentials. In A. G. Karczmar \& J. C. Eccles (Eds.), Brain and human behavior. New York: SpringerVerlag, 1972. Pp. 303-323.

Moruzzi, G. Reticular influences on the EEG. Electroencephalography and Clinical Neurophysiology, 1964, 16, 2-17.

MoruzzI, G., \& MAGoun, H. W. Brain stem reticular formation and activation of the EEG. Electroencephalography and Clinical Neurophysiology, 1949, 1, 455-473.

OLDs, J. Mechanisms of instrumental conditioning. Electroencephalography and Clinical Neurophysiology, 1963, Supp. 24.

Olds, J., \& Olds, M. E. Approach-avoidance analysis of rat diencephalon. Journal of Comparative Neurology, 1963, 120. 259-295.

Rosenfeld, J. P. Evoked potential conditioning in neuroscience research. In M. H. Chase (Ed.), Operant control of brain activity. Los Angeles: Brain Information Service, 1974. Pp. 105-144.

Routtenberg, A., \& Malsbury, C. Brainstem pathways of reward. Journal of Comparative and Physiological Psychology, 1969, 68, 22-30.

SkInNer, J. E. Neuroscience: A laboratory manual. Philadelphia: Saunders, 1971.

(Received for publication September 30, 1976; revision accepted December $10,1976$. ) 